\title{
Phonons and solitons in the "thermal" sine-Gordon system
}

\author{
Salerno, Mario; Jørgensen, E.; Samuelsen, Mogens Rugholm
}

Published in:

Physical Review B

Link to article, DOI:

10.1103/PhysRevB.30.2635

Publication date:

1984

Document Version

Publisher's PDF, also known as Version of record

Link back to DTU Orbit

Citation (APA):

Salerno, M., Jørgensen, E., \& Samuelsen, M. R. (1984). Phonons and solitons in the "thermal" sine-Gordon system. Physical Review B, 30(5), 2635-2639. https://doi.org/10.1103/PhysRevB.30.2635

\section{General rights}

Copyright and moral rights for the publications made accessible in the public portal are retained by the authors and/or other copyright owners and it is a condition of accessing publications that users recognise and abide by the legal requirements associated with these rights.

- Users may download and print one copy of any publication from the public portal for the purpose of private study or research.

- You may not further distribute the material or use it for any profit-making activity or commercial gain

- You may freely distribute the URL identifying the publication in the public portal

If you believe that this document breaches copyright please contact us providing details, and we will remove access to the work immediately and investigate your claim 


\title{
Phonons and solitons in the "thermal" sine-Gordon system
}

\author{
Mario Salerno* \\ Laboratory of Applied Mathematical Physics, The Technical University of Denmark, DK-2800 Lyngby, Denmark \\ E. Joergensen and M. R. Samuelsen \\ Physics Laboratory I, The Technical University of Denmark, DK-2800 Lyngby, Denmark
}

(Received 23 September 1983)

\begin{abstract}
Standard methods of stochastic processes are used to study the coupling of the sine-Gordon system with a heat reservoir. As a result we find thermal phonons with an average energy of $k_{B} T$ per mode. The translational mode (zero mode) is found to carry an average energy of $\frac{1}{2} k_{B} T$. This last value is just the energy in the Brownian motion of a thermal soliton. These results are in agreement with those obtained by the use of a statistical-mechanical description of a dilute soliton gas. Connection of the above results with Josephson junctions and the extension of the analysis to more general equations is also discussed.
\end{abstract}

\section{INTRODUCTION}

The sine-Gordon equation has recently been used to model several physical systems in contact with a heat reservoir at a given temperature. ${ }^{1-9}$ The effect of the coupling of the sine-Gordon system to the heat reservoir provides a twofold mechanism:

(i) a dissipation of energy in the system due to an energy flow from the system to the heat reservoir,

(ii) a disordered input of energy into the system due to a flow back of energy from the reservoir.

A loss term in the sine-Gordon equation is then intrinsically connected to a thermal noise term, suggesting a modeling of the interaction between the system and the reservoir with a driving stochastic force (temperature dependent) in the pure sine-Gordon equation: ${ }^{1,3-5,7}$

$$
\phi_{x x}-\phi_{t t}-\sin \phi=\alpha \phi_{t}+n(x, t) .
$$

The first term on the right-hand side (rhs) of Eq. (1.1) is the loss term representing the energy flow to the reservoir, while the second term is the noise associated with $\alpha$, giving the disordered thermal-energy input to the system. The noise term is assumed to be "white" both in space and time with the autocorrelation function:

$$
\left\langle n(x, t) n\left(x^{\prime}, t^{\prime}\right)\right\rangle=16 \alpha\left(k_{B} T / E_{0}\right) \delta\left(x-x^{\prime}\right) \delta\left(t-t^{\prime}\right) .
$$

Here $\langle\cdots\rangle$ means ensemble average, while the constant $16 \alpha\left(k_{B} T / E_{0}\right)$ is determined by applying the fluctuation dissipation theorem for a soliton with small velocity $1,4,7,10$ ( $E_{0}$ is the rest energy of a soliton and is used to fix the scale of energy in the system, $k_{B}$ is the Boltzmann constant, and $T$ is the temperature).

When $\alpha=0, n(x, t)=0$, Eq. (1.1) reduces to the pure sine-Gordon equation with the exact soliton solution

$$
\phi=4 \tan ^{-1}\left[\exp \pm\left[\frac{x-v t}{\left(1-v^{2}\right)^{1 / 2}}\right]\right]
$$

and Hamiltonian density

$$
H=\frac{E_{0}}{8}\left[\frac{1}{2}\left(\phi_{x}^{2}+\phi_{t}^{2}\right)+1-\cos \phi\right] .
$$

Small oscillations around a ground state $\phi_{0}$ of the system are obtained by linearizing the sine-Gordon equation with

$$
\phi=\phi_{0}+\psi,
$$

this providing a linear equation for $\psi$ :

$$
\psi_{x x}-\psi_{t t}-\psi \cos \phi_{0}=0,
$$

with an associated energy density given by

$$
H_{\mathrm{ph}}=\frac{E_{0}}{16}\left(\psi_{x}^{2}+\psi_{t}^{2}+\psi^{2} \cos \phi_{0}\right) \text {. }
$$

When the ground state of the system is given by (1.3), a "zero mode" (translational mode) is found from Eq. (1.6). In addition to this mode, there exists a continuum set of states (phonon modes) which satisfy the linear dispersion relation: ${ }^{11}$

$$
\omega^{2}=1+k^{2} \text {. }
$$

For practical applications to Josephson junctions, it is of interest to include also in the rhs of Eq. (1.1) a constant bias term $\eta$, representing an ordered energy input into the system (work on the system). In this case (in the absence of solitons), phonons (also called "plasmons") are seen as small oscillations around the ground state $\phi_{0}$ $=-\sin ^{-1} \eta .^{12}$

In this paper we study the effect of the heat reservoir both on solitons and phonons by using standard methods of stochastic processes. This will be done in the following cases.

In Sec. II we study thermal phonons in the presence of a static "exact" sine-Gordon soliton. In Sec. III we include a $\eta$ bias term in the rhs of (1.1) and study the thermally excited plasmons around $\phi_{0}=-\sin ^{-1} \eta$ (no solitons present in the system).

In both cases we find that as long as $k_{B} T \ll<E_{0}$, the 
phonon modes have an average energy of $k_{B} T$ per mode. In the case when a soliton is in the system, however, we find that the corresponding extra mode (zero mode), has an average energy of $\frac{1}{2} k_{B} T$. All these results are in agreement with a statistical-mechanical description of the sine-Gordon system. In Sec. IV we concentrate on the effect of both the bias term $(\eta)$ and the heat reservoir [ $\alpha$ and $n(x, t)]$ on the soliton motion. As result of the "thermalization" the soliton will execute a Brownian motion with average energy of $\frac{1}{2} k_{B} T$ (the zero-mode energy). In Sec. $\mathrm{V}$ we relate the above results to a practical Josephson junction and, finally, in Sec. VI we give a short summary of the main results of the paper including a brief discussion of the possibility of extending the analysis to other equations of the nonlinear Klein-Gordon class.

\section{THERMAL PHONONS IN THE PRESENCE OF A SOLITON}

We consider as an "unthermalized" system the pure sine-Gordon equation

$$
\phi_{x x}-\phi_{t t}-\sin \phi=0
$$

and assume that only a static soliton is present (dilute-gas limit). Phonon modes $\psi_{k}$ are obtained as solutions of Eq. (1.6) with $\phi_{0}$ given by (1.3) with $v=0$. Assuming $\psi_{k}$ as

$$
\psi_{k}(x, t)=f_{k}(x) e^{i \omega_{k} t},
$$

we obtain from Eq. (1.6) that

$$
\left(-\partial_{x x}+1-2 \operatorname{sech}^{2} x\right) f_{k}(x)=\omega_{k}^{2} f_{k}(x) .
$$

As is well known, (2.3) admits a continuum set of eigenfunctions:

$$
f_{k}(x)=\frac{1}{(2 \pi)^{1 / 2}} e^{i k x}\left(1+k^{2}\right)^{-1 / 2}(k+i \tanh x)
$$

together with a zero mode:

$$
f_{b}(x)=\frac{1}{\sqrt{2}} \operatorname{sech} x
$$

which restores the translational symmetry broken by the introduction of the soliton into the system (Goldstone mode). ${ }^{11}$ Equations (2.4) and (2.5) together form a complete set of orthonormal eigenfunctions:

$$
\begin{aligned}
& \int_{-\infty}^{+\infty} f_{b}^{2}(x) d x=1, \quad \int_{-\infty}^{+\infty} f_{b}(x) f_{k}(x) d x=0 \\
& \int_{-\infty}^{+\infty} f_{k}^{*}(x) f_{k^{\prime}}(x) d x=\delta\left(k-k^{\prime}\right) \\
& f_{b}(x) f_{b}\left(x^{\prime}\right)+\int_{-\infty}^{+\infty} f_{k}^{*}(x) f_{k}\left(x^{\prime}\right) d k=\delta\left(x-x^{\prime}\right)
\end{aligned}
$$

where $*$ in the superscript means complex conjugate.

By coupling the sine-Gordon system with the heat reservoir, we change Eq. (2.1) into Eq. (1.1). Thermal phonons are then found to satisfy

$$
\psi_{x x}-\psi_{t t}-\psi \cos \phi_{0}=\alpha \psi_{t}+n(x, t)
$$

for which the general solution can be expanded in terms of the complete set (2.6) as

$$
\psi_{k}(x, t)=\sum_{n=0}^{\infty} A_{k}(t) f_{k}(x)+A_{b}(t) f_{b}(x)
$$

(here we assume the system to be in a box of length $L$, and then let $L \rightarrow \infty)$. Substituting (2.8) in (2.7) and using Eq. (2.3), we obtain

$$
\begin{gathered}
\sum_{n=0}^{\infty}\left[A_{k, t t} f_{k}(x)+\alpha A_{k, t} f_{k}(x)+A_{k} \omega_{k}^{2} f_{k}(x)\right] \\
+\left(A_{b, t t}+\alpha A_{b, t}\right) f_{b}(x)=-n(x, t) .
\end{gathered}
$$

Equation (2.9) is easily studied once projected, respectively, along the $f_{k}(x)$ 's and the $f_{b}(x)$ eigenfunctions, this giving [using (2.6)]

$$
A_{k, t t}+\alpha A_{k, t}+A_{k} \omega_{k}^{2}=\epsilon_{k}(t)
$$

and

$$
A_{b, t t}+\alpha A_{b, t}=\epsilon_{b}(t)
$$

with

$$
\begin{aligned}
& \epsilon_{b}(t)=-\int_{-\infty}^{+\infty} f_{b}(x) n(x, t) d x, \\
& \epsilon_{k}(t)=-\int_{-\infty}^{+\infty} f_{k}^{*}(x) n(x, t) d x .
\end{aligned}
$$

By using (1.2) and (2.6) we find, for the autocorrelation function $R_{\epsilon}\left(t-t^{\prime}\right)$ and for the power spectrum $S_{\epsilon}(\omega)$ of the normal processes $\epsilon_{k}(t)$ and $\epsilon_{b}(t)$, that

$$
\begin{aligned}
& R_{\epsilon_{k}}\left(t-t^{\prime}\right)=R_{\epsilon_{b}}\left(t-t^{\prime}\right) \\
& =16 \alpha\left(k_{B} T / E_{0}\right) \delta\left(t-t^{\prime}\right), \\
& S_{\epsilon_{k}}(\omega)=S_{\epsilon_{b}}(\omega)=16 \alpha\left(k_{B} T / E_{0}\right) .
\end{aligned}
$$

Equations (2.10) and (2.11) are then integrated by the standard theory of stochastic processes, ${ }^{10}$ giving the following expressions for the power spectrum of $A_{k}(t)$ and $A_{k, t}(t)$ :

$$
\begin{aligned}
& S_{A_{k}}(\omega)=16 \alpha\left(k_{B} T / E_{0}\right) \frac{1}{\left(\omega^{2}-\omega_{k}^{2}\right)^{2}+\alpha^{2} \omega^{2}}, \\
& S_{A_{k, t}}(\omega)=\omega^{2} S_{A_{k}}(\omega) .
\end{aligned}
$$

[ $S_{A_{b}}(\omega)$ and $S_{A_{b, t}}(\omega)$ are obtained from (2.14) and (2.15) with the substitution $\omega_{k}=0$.] If we assume ergodicity, the time averages of the processes $\left|A_{k}(t)\right|^{2}$ and $\left|A_{k, t}(t)\right|^{2}$ are evaluated as

$$
\begin{aligned}
\left\langle\left|A_{k}(t)\right|^{2}\right\rangle & =R_{A_{k}}(0) \\
& =\int_{-\infty}^{+\infty} \frac{d \omega}{2 \pi} S_{A_{k}}(\omega)=8\left(k_{B} T / \omega_{k}^{2} E_{0}\right), \\
\left\langle\left|A_{k, t}(t)\right|^{2}\right\rangle & =R_{A_{k, t}}(0) \\
& =\int_{-\infty}^{+\infty} \frac{d \omega}{2 \pi} S_{A_{k, t}}(\omega)=8\left(k_{B} T / E_{0}\right),
\end{aligned}
$$

where contour integration has been used in evaluating the integrals in (2.16) and (2.17). In the same way we obtain 


$$
\text { for } \begin{aligned}
& \left\langle\left|A_{b, t}(t)\right|^{2}\right\rangle \\
& \left\langle\left|A_{b, t}(t)\right|^{2}\right\rangle=8\left(k_{B} T / E_{0}\right) .
\end{aligned}
$$

From (1.7) and (2.8) we have that the average energies of the $k$ th phonon mode $A_{k}(t) f_{k}(x)$ and of the translational mode $A_{b}(t) f_{b}(x)$ are given, respectively, by

$$
\left\langle H_{k}\right\rangle=\frac{E_{0}}{16}\left[\left\langle\left|A_{k, t}(t)\right|^{2}\right\rangle+\omega_{k}^{2}\left\langle\left|A_{k}(t)\right|^{2}\right\rangle\right]
$$

and

$$
\left\langle H_{b}\right\rangle=\frac{E_{0}}{16}\left\langle\left|A_{b, t}\right|^{2}\right\rangle .
$$

Using (2.16)-(2.18), we finally have

$$
\left\langle H_{k}\right\rangle=k_{B} T
$$

and

$$
\left\langle H_{b}\right\rangle=\frac{1}{2} k_{B} T
$$

in complete agreement with the classical statisticalmechanics analysis of a dilute soliton gas derived in Ref. 8.

\section{THERMAL PHONONS IN THE ABSENCE OF SOLITONS WITH BIAS}

In this section we consider the unthermalized system to be the pure sine-Gordon system of finite length $L$, with a constant driving force $\eta<1$ :

$$
\phi_{x x}-\phi_{t t}-\sin \phi=\eta \text {. }
$$

Phonon modes $\psi_{n}$ are seen as small oscillations around the classical ground state

$$
\phi_{0}=-\sin ^{-1} \eta,
$$

satisfying the boundary conditions

$$
\psi_{n, x}(0, t)=\psi_{n, x}(L, t)=0
$$

(no solitons are present in the system). The thermalized system is obtained from Eq. (3.1) by adding to the rhs the term $\alpha \phi_{t}+n(x, t)$ with $n(x, t)$ given as in (1.2):

$$
\phi_{x x}-\phi_{t t}-\sin \phi=\eta+\alpha \phi_{t}+n(x, t) .
$$

Thermal phonons are then solutions of the following stochastic equation:

$$
\psi_{x x}=\psi_{t t}-\left(1-\eta^{2}\right)^{1 / 2} \psi=\alpha \psi_{t}+n(x, t) .
$$

When $\alpha=0, n(x, t)=0$, these phonons are just classical Klein-Gordon modes with energy given by

$$
H_{\mathrm{ph}}=\frac{E_{0}}{16} \int_{0}^{L} d x\left[\psi_{x}^{2}+\psi_{t}^{2}+\psi^{2}\left(1-\eta^{2}\right)^{1 / 2}\right]
$$

The general solution of Eq. (3.5) satisfying the boundary conditions (3.3) is of the form

$$
\psi=(2 / L)^{1 / 2} \sum_{n} A_{n}(t) \cos \left(k_{n} x\right)
$$

with

$$
k_{n}=\frac{n \pi}{L}
$$

and $(2 / L)^{1 / 2}$ being just a normalization factor (for $n=0$ it should be read as $L^{-1 / 2}$ ). Substituting (3.7) in (3.5) and applying to both sides of the equation the projection operator $\int_{0}^{L} \cos \left(k_{n} x\right) d x$, we obtain

$$
A_{n, t t}+\alpha A_{n, t}+\left[\left(1-\eta^{2}\right)^{1 / 2}+k_{n}^{2}\right] A_{n}=\epsilon_{n}(t),
$$

where

$$
\epsilon_{n}(t)=(2 / L)^{1 / 2} \int_{0}^{L} n(x, t) \cos \left(k_{n} x\right) d x .
$$

Using (1.2), we find for the autocorrelation function $R_{\epsilon_{n}}\left(t-t^{\prime}\right)$ and the power spectrum $S_{\epsilon_{n}}(\omega)$ the same expression as in (2.12) and (2.13). By identifying $\left[\left(1-\eta^{2}\right)^{1 / 2}+k_{n}^{2}\right]$ with $\omega_{n}^{2}$, we see that Eq. (3.9) in the limit $L \rightarrow \infty$ coincides with Eq. (2.0), and therefore, following the same analysis of the preceding section, we obtain that the average energy per phonon mode is

$$
\left\langle H_{n}\right\rangle=k_{B} T \text {. }
$$

No zero-mode energy is present in this case, due to the absence of the soliton in the system. Finally, we remark that the above results do not depend on the particular boundary condition (3.3) used (we could have used generic periodic boundary conditions) as well as on smallness requirements of $\alpha$ and $\eta$. The only approximation that has been made in obtaining (2.21), (2.22), and (3.11) is the linearization procedure, which is justified if

$$
k_{B} T \ll E_{0},
$$

as appears evident from Eqs. (2.16) and (2.17).

\section{BROWNIAN MOTION AND DIFFUSION CONSTANT OF A THERMAL SOLITON}

We now concentrate on the effect of the $\alpha, \eta$, and $n(x, t)$ terms in Eq. (3.4) on the soliton motion (here a soliton is a $2 \pi$-kink jump from $-\sin ^{-1} \eta$ to $2 \pi-\sin ^{-1} \eta$ ). We assume $\eta / \alpha$ and $k_{B} T / E_{0}$ to be small. By introducing the momentum

$$
P=-\frac{1}{8} \int_{-\infty}^{+\infty} \phi_{x} \phi_{t} d x,
$$

and differentiating with respect to time, we obtain

$$
\frac{d P}{d t}=-\alpha P+\frac{1}{4} \pi \eta+\epsilon(t),
$$

where we have used Eq. (3.4) to eliminate the $\phi_{t t}$ term and have defined $\epsilon(t)$ as

$$
\epsilon(t)=\frac{1}{8} \int_{-\infty}^{+\infty} \phi_{x}(x, t) n(x, t) d x .
$$

Neglecting the noise term, Eq. (4.2) describes the "power balance" motion of a $2 \pi$-kink with velocity ${ }^{13}$

$$
u_{0}= \pm\left[1+\left[\frac{4 \alpha}{\pi \eta}\right]^{2}\right]^{-1 / 2}
$$


and momentum

$$
P_{0}=\frac{u_{0}}{\left(1-u_{0}^{2}\right)^{1 / 2}}
$$

The noise term $n(x, t)$ in Eq. (4.2) introduces fluctuations in the momentum and, from (4.5), in the velocity of the kink. Such fluctuations are readily evaluated by standard techniques (for details see Ref. 7), giving the following for the power spectrum of the process $\Delta u(t)=u(t)-u_{0}$ :

$$
S_{\Delta u}(\omega)=2 \alpha\left(k_{B} T / E_{0}\right) \frac{\left(1-u_{0}^{2}\right)^{5 / 2}}{\omega^{2}+\alpha^{2}} .
$$

By assuming $u_{0} \ll<$, Eq. (4.6) reduces to

$$
S_{u}(\omega)=2 \alpha\left(k_{B} T / E_{0}\right) \frac{1}{\omega^{2}+\alpha^{2}}
$$

from which we obtain

$$
\left\langle u^{2}\right\rangle=\int_{-\infty}^{+\infty} \frac{d \omega}{2 \pi} S_{u}(\omega)=\left(k_{B} T / E_{0}\right) .
$$

The time average of the kinetic energy in the Brownian motion of the soliton is then given by

$$
\left\langle E_{\text {sol }}\right\rangle=\frac{1}{2} E_{0}\left\langle u^{2}\right\rangle=\frac{1}{2} k_{B} T
$$

as expected from soliton statistical-mechanics theory. ${ }^{8}$

Finally, from Eq. (4.6) a diffusion constant $D$ for the $2 \pi$-kink motion is derived:

$$
D=\frac{1}{E_{0}}\left(k_{B} T / \alpha\right),
$$

which is just the usual Einstein diffusion constant for the Brownian motion of a particle in a viscous medium (this is a further confirmation of the particlelike nature of the soliton). (See also Ref. 3.)

\section{THERMAL SOLITONS AND PHONONS IN JOSEPHSON JUNCTIONS}

We will now relate the foregoing sections to a real device as the Josephson junction. We will find the orders of magnitude of the quantities of interest and see if the assumption made in the above analysis holds for Josephson junctions.

The fluxon-rest energy (in laboratory units) for a Josephson junction is

$$
E_{0}=\hbar \omega_{0}=8 \hbar \lambda_{J} J L /(2 e),
$$

where $J$ is the maximum Josephson current density, $L$ is the length of the junction, and $e$ is the electron charge. $\lambda_{J}$ in (5.1) is the Josephson penetration depth given by

$$
\lambda_{J}=\left(\hbar / 2 e \mu_{0} d J\right)^{1 / 2}
$$

where $d$ is the magnetic thickness of the oxide layer $\left(2 \lambda_{L}+t_{0}\right)$, and $\mu_{0}$ is the vacuum permeability. From (5.1) we have that for a typical Josephson junction

$$
\left(k_{B} T / E_{0}\right) \approx 10^{-4}-10^{-5},
$$

which justifies the assumption (3.12) made in the analysis.
The Josephson plasma frequency is

$$
\omega_{P}=\left(2 e J t_{0} / \epsilon_{0} \epsilon_{r} \hbar\right)^{1 / 2},
$$

where $\epsilon_{r}$ and $\epsilon_{0}$ are, respectively, the relative dielectric constant of the oxide layer and the dielectric constant of the vacuum, while $t_{0}$ is the thickness of the oxide layer. For a plasmon described in Sec. III we have that the splitting of the energy level is

$$
E_{\mathrm{pl}}^{q}=\hbar \omega_{p} \omega_{n}
$$

with $\omega_{n}$ given by $\left[\left(1-\eta^{2}\right)^{1 / 2}+(n \pi / L)^{2}\right]^{1 / 2}$. We have then that the ratio $k_{B} T / E_{\mathrm{pl}}^{q}$ is of the order of magnitude $1-10$, i.e., the quantum energy levels are separated by a quantity comparable with $k_{B} T$. To have a rough estimate of the energy-level separation for a fluxon, we can use the analogy of a particle in a box. This gives

$$
E_{\mathrm{fl}}^{q}=\frac{\pi^{2} \hbar^{2}}{2 M_{0}} \frac{n^{2}}{L^{2}} \approx 10^{-4} \hbar \omega_{p},
$$

.e., for a fluxon the separation in the energy levels is smaller than $k_{B} T$ by a factor of the order $10^{-3}-10^{-4}$. This numerical manipulation indicates that for a typical Josephson junction fluxon quantitation is not necessary, while it is necessary for plasmons ( $E_{\mathrm{pl}}^{q}$ being of the same order of magnitude as $k_{B} T$ ). In Ref. 6 the effects of quantum plasmons on the fluxon motion have been calculated. It turns out that they are several orders of magnitude smaller than the direct influence of the thermal reservoir on the soliton evaluated in this paper, and therefore, in our context, completely negligible.

Finally, in closing this section it is worth noting that if $k_{B} T / E_{0}$ is very small, a statistical-mechanical description of fluxons in Josephson junctions is meaningless. However, the method used in the preceding section is still useful to study the interactions between plasmons and fluxons. (See Ref. 7 for the case of Josephson oscillators.)

\section{CONCLUSION}

It has been shown that the effect of a thermal reservoir on the sine-Gordon system can be studied by using standard methods of stochastic processes. Both phonons and solitons are found to be thermalized in a way that the phonons will have an average energy of $k_{B} T$ per mode, while solitons will have an energy of $\frac{1}{2} k_{B} T$. These results are in agreement with those obtained by using a statistical-mechanics approach for a "dilute" solution gas. $^{8}$ The main assumption used in our derivation has been $k_{B} T \ll E_{0}$ (to justify the linearization procedure). Second-order effects [in the small quantity $\left(k_{B} T / E_{0}\right)$ ], such as interaction between phonon modes and solitons, ${ }^{6,9}$ have been neglected therefore. Finally, in closing this paper we wish to point out that in spite of the particularity of the model used, the results obtained are sufficiently general to be extended to other equations of the nonlinear Klein-Gordon class, such as $\phi^{4}$, double sine-Gordon, etc. As a matter of fact, the only difference in the analysis will be the presence of additional bound states in the linear phonon eigenvalue problem. By following arguments 
similar to those used in the present paper, however, it is easily shown that each of these additional bound states carries a thermal-average energy of $k_{B} T$. This energy will not increase the energy of the center of mass of a solitonlike solition of these more general models, but it will increase the energy of the internal degrees of freedom (motion around the center of mass) of these excitations. ${ }^{14}$

\section{ACKNOWLEDGMENTS}

One of us (M. S.) would like to thank the Laboratory of Applied Mathematical Physics (LAMF) (where part of this paper was done), for the kind hospitality received, the Danish Ministry of Education, and the European Research Office of the United States Army (Contract No. DAJA-37-82-C-0057) for providing financial support.
*Permanent address: Istituto di Fisica, Università degli Studi di Salerno, I-84100 Salerno, Italy.

${ }^{1}$ S. E. Trullinger, M. D. Miller, R. A. Guyer, A. R. Bishop, F. Palmer, and J. A. Krumhansl, Phys. Rev. Lett. 40, 206 (1978).

${ }^{2}$ H. J. Mikeska, J. Phys. C 11, 129 (1978); K. Maki, J. Low Temp. Phys. 41, 327 (1980); D. J. Bergman, E. Ben Jacob, Y. Imry, and K. Maki, Phys. Rev. A 27, 3345 (1983).

${ }^{3}$ M. Reimossenet, Solid State Commun. 27, 681 (1978); L. Gunther and Y. Imry, Phys. Rev. Lett. 44, 1225 (1980).

${ }^{4}$ M. Büttiker and R. Landauer, Phys. Rev. A 23, 1397 (1981).

${ }^{5}$ D. J. Kaup, Phys. Rev. B 27, 6787 (1983).

${ }^{6}$ M. Salerno and A. C. Scott, Phys. Rev. B 26, 2474 (1982).
${ }^{7}$ E. Joergensen, V. P. Koshelets, R. Monaco, J. Mygind, M. R. Samuelsen, and M. Salerno, Phys. Rev. Lett. 49, 1093 (1982).

${ }^{8}$ A. R. Bishop, J. A. Krumhansl, and S. E. Trullinger, Physica (Utrecht) 1D, 1 (1980).

${ }^{9}$ Y. Wada and H. Ishiuchi, J. Phys. Soc. Jpn. 51, 1372 (1982).

${ }^{10}$ See, for example, A. Papoulis, Probability, Random Variables, and Stochastic Processes (McGraw-Hill, New York, 1965).

11J. Rubinstein, J. Math. Phys. 11, 258 (1970).

${ }^{12}$ O. H. Olsen and M. R. Samuelsen, Phys. Rev. B 28, 210 (1983).

${ }^{13}$ D. W. McLaughlin and A. C. Scott, Phys. Rev. A 18, 1652 (1978).

${ }^{14}$ M. Salerno and M. R. Samuelsen (unpublished). 